\title{
Optimal Power Allocation for MIMO-OFDM Based Cognitive Radio Systems with Arbitrary Input Distributions
}

\author{
Ahmed Sohail, Mohammed Al-Imari, Pei Xiao, Barry G. Evans \\ Centre for Communication Systems Research, University of Surrey, Guildford, GU2 7XH, UK \\ Email: \{a.sohail,m.al-imari,p.xiao,b.evans\}@ surrey.ac.uk
}

\begin{abstract}
In Cognitive Radio (CR) systems, the data rate of the Secondary User (SU) can be maximized by optimizing the transmit power, given a threshold for the interference caused to the Primary User (PU). In conventional power optimization algorithms, the Gaussian input distribution is assumed, which is unrealistic, whereas the Finite Symbol Alphabet (FSA) input distribution, (i.e., M-QAM) is more applicable to practical systems. In this paper, we consider the power optimization problem in multiple input multiple output orthogonal frequency division multiplexing based CR systems given FSA inputs, and derive an optimal power allocation scheme by capitalizing on the relationship between mutual information and minimum mean square error. The proposed scheme is shown to save transmit power compared to its conventional counterpart. Furthermore, our proposed scheme achieves higher data rate compared to the Gaussian optimized power due to fewer number of subcarriers being nulled. The proposed optimal power algorithm is evaluated and compared with the conventional power allocation algorithms using Monte Carlo simulations. Numerical results reveal that, for distances between the $S U$ transmitter and the $P U$ receiver ranging between $50 \mathrm{~m}$ to $85 \mathrm{~m}$, the transmit power saving with the proposed algorithm is in the range $13-90 \%$, whereas the rate gain is in the range $5-31 \%$ depending on the modulation scheme (i.e., BPSK, QPSK and 16-QAM) used.
\end{abstract}

Index Terms-Cognitive Radio, OFDM, MIMO, Finite Symbol Alphabet, MMSE, Mutual Information.

\section{INTRODUCTION}

The current static frequency band allocations lead to poor spectrum utilization and encourage the regulatory bodies to review their spectrum allocation and encompass more sharing and dynamic allocations. Spectrum occupancy measurements conducted by Ofcom [1] in different areas of the UK, show underutilization of spectrum for significant periods of time. Similarly, the FCC [2], in New York City and downtown Washington DC show only $13.1 \%$ and $35 \%$ of spectrum utilization, respectively below $3 \mathrm{GHz}$. These studies clearly suggest that physical spectrum shortage is mainly due to inflexible spectrum licensing schemes. This gave rise to the development of Cognitive Radio (CR) system, which allows the Secondary User (SU) to opportunistically access the licensed spectrum given acceptable interference to the Primary User (PU) [3]. The following spectrum sharing schemes have been presented for CR systems in [4]: underlay spectrum sharing, overlay spectrum sharing and Interweave (opportunistic) Spectrum Sharing (ISS). The ISS scheme is preferred due to its ability to achieve higher data rates as it allows the SU to opportunistically access the PU band. It is therefore the focus of our study in this paper.

In current wireless communication standards and services, Orthogonal Frequency Division Multiplexing (OFDM) is widely used due to its mitigation of the multipath propagation problem [5]. It is also very suitable for CR systems due to its ability to monitor the PU spectral activity and having the flexibility to dynamically allocate unused spectrum among the SU subcarriers [6].

Recent research on resource allocation in CR system assumes Single Input Single Output (SISO) techniques, however, in todays systems, available resources (e.g., bandwidth, transmit power etc.) are limited. Therefore, Multiple Input Multiple Output (MIMO) techniques have been introduced to increase the capacity without requiring additional bandwidth and power compared to the SISO technique. MIMO can also provide more degrees of freedom to the $\mathrm{SU}$ in order to balance between achievable rate and interference introduced to the PU. Furthermore, combining MIMO with OFDM is regarded as a very attractive solution for CR systems to effectively enhance channel capacity over multipath fading channels compared to SISO-OFDM. However, the role of MIMO in CR system remains to be exploited.

In opportunistic spectrum access, where the PU and the SU co-exist in adjacent bands, mutual interference (i.e., from SU to PU and vice versa) is the limiting factor on performance of both networks. Power allocation in OFDM based CR systems aims to dynamically control the transmit power on each subcarrier of the SU in order to reduce the mutual interference. Traditional power allocation schemes, i.e., water-filling etc. cause more interference in the CR scenario, hence, a judicious power allocation scheme is required which takes into consideration the channel condition as well as the spectral distance between the SU's subcarriers and the PU. Different power allocation schemes have been proposed in the literature [7], [8] where Gaussian inputs are assumed to maximize the SU data rate for a given interference threshold value. However, the Gaussian assumption does not match practical and more accurately a Finite Symbol Alphabet (FSA) input is more applicable to practical systems. To determine the difference between the Gaussian and the FSA input, a Signal-to-Noise Ratio (SNR) gap model has been proposed in [9], where the achievable rates attained by the FSA input are approximated 
by the capacity attained by the Gaussian input. However, this approach is not valid at high SNRs due to the large gap and its inability to predict the rate saturation point. In [10], a mercury water-filling algorithm is proposed in order to derive optimal power allocation using the FSA input. However, in this work, authors considered a non-cognitive scenario, whereas in interference limited CR systems, the same mercury waterfilling algorithm cannot be applied due to mutual interference, which degrades the performance of both PU and SU networks. Therefore, in [11], we derived the optimal power in OFDM based CR systems given an FSA input distribution.

The aforementioned work addresses power allocation algorithms in SISO-OFDM based CR systems. In [12], optimal power is evaluated for MIMO-OFDM based CR systems but again with the Gaussian input assumption. To the best of our knowledge, no work has been done to derive and evaluate optimal power with arbitrary input distributions in MIMOOFDM based CR systems. The contributions of this paper are summarized as follows;

- We propose to formulate a convex optimization problem and derive the optimal power allocation for an FSA input distribution by capitalizing on the relationship between Mutual Information (MI) and Minimum Mean Square Error (MMSE) [13].

- We show that if the conventionally optimized power with the Gaussian input assumption is used for the FSA transmission, there will be a wastage of transmit power; whereas the optimal power allocation derived by the proposed scheme leads to a significant power saving. Moreover, the conventional scheme also results in a reduced transmission rate due to the fact that extra allocated power causes nulling of more subcarriers compared to the proposed scheme.

The remainder of the paper is organized as follows. Sections II and III present the system model and optimal power allocation policy for MIMO-OFDM based CR systems, respectively. We present simulation results of the proposed scheme in Section IV. Finally, conclusions are drawn in Section V.

\section{SySTEM MODEL}

The system model consists of a single-cell wireless system in the downlink, where the PU and the SU transceivers coexist in the same geographical location as shown in Fig. 1. We consider the co-existence of a PU and a SU in the frequency domain where the user data are mapped to consecutive subcarriers as shown in Fig. 2. It is assumed that the SU employs OFDM modulation and has $L_{t}$ transmit antennas and $L_{r}$ receive antennas. Similarly, the PU has $M_{r}$ receive antennas. The MIMO channel for $n$th subcarrier between the SU transmitter and receiver is denoted by $\mathbf{H}_{n} \in \mathcal{C}^{L_{r} \times L_{t}}$. The received vector $\mathbf{y}_{n} \in \mathcal{C}^{L_{r} \times 1}$ for $n$th subcarrier corresponding to the transmit vector $\mathbf{x}_{n} \in \mathcal{C}^{L_{t} \times 1}$ is given as

$$
\mathbf{y}_{n}=\mathbf{H}_{n} \mathbf{x}_{n}+\mathbf{z}_{n},
$$

where $\mathbf{z}_{n} \in \mathcal{C}^{L_{r} \times 1}$ is the additive white Gaussian noise vector. Here $\mathbf{x}_{n}=\left[\sqrt{p_{n 1}} b_{n 1}, \ldots, \sqrt{p_{n L_{t}}} b_{n L_{t}}\right]$, where $p_{n \ell_{t}}, b_{n \ell_{t}}$ are

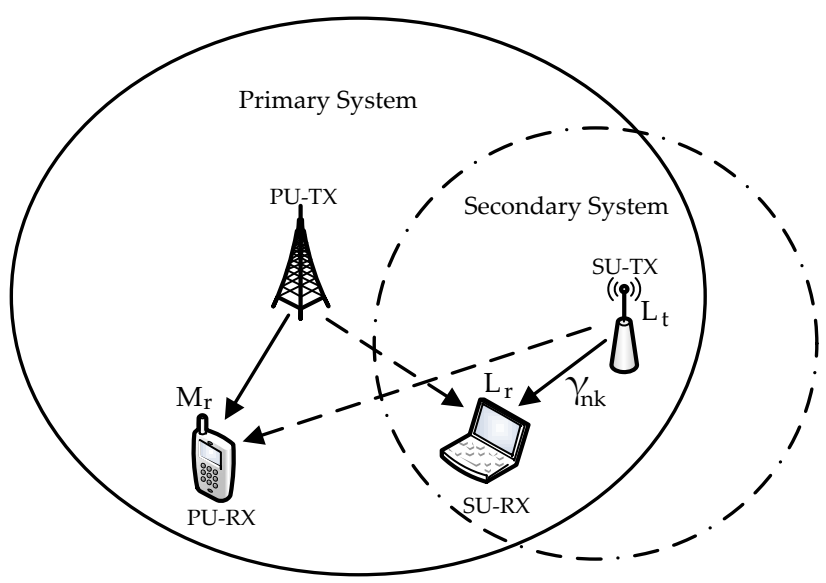

Fig. 1. Distribution of PU and SU for MIMO CR System.

the transmit power and unit power symbols of the $n$th subcarrier at the $\ell_{t}$ th antenna, respectively. The Singular Value Decomposition (SVD) of the channel matrix $\mathbf{H}_{n}$ is given as

$$
\mathbf{H}_{n}=\mathbf{U}_{n} \boldsymbol{\Sigma}_{n} \mathbf{V}_{n}^{*},
$$

where $\mathbf{U}_{n} \in \mathcal{C}^{L_{r} \times L_{r}}$ and $\mathbf{V}_{n}^{*} \in \mathcal{C}^{L_{t} \times L_{t}}$ are unitary matrices and $\boldsymbol{\Sigma}_{n} \in \mathcal{C}^{L_{r} \times L_{t}}$ is the diagonal matrix containing non-negative ordered eigenvalues of $\mathbf{H}_{n} \mathbf{H}_{n}^{*}$, i.e., $\gamma_{n 1} \geq$ $, \ldots, \gamma_{n K} \geq 0$. Let $K$ denote the number of eigenvalues, where $K=\min \left(L_{r}, L_{t}\right)$. The columns of $\mathbf{U}_{n}$ are the eigenvectors of $\mathbf{H}_{n} \mathbf{H}_{n}^{*}$ and the columns of $\mathbf{V}_{n}$ are eigenvectors of $\mathbf{H}_{n}^{*} \mathbf{H}_{n}$. Thus, Eq.(1) becomes

$$
\mathbf{y}_{n}=\mathbf{U}_{n} \boldsymbol{\Sigma}_{n} \mathbf{V}_{n}^{*} \mathbf{x}_{n}+\mathbf{z}_{n} .
$$

Let $\widetilde{\mathbf{y}}_{n}=\mathbf{U}_{n}^{*} \mathbf{y}_{n}, \widetilde{\mathbf{x}}_{n}=\mathbf{V}_{n} \mathbf{x}_{n}$ and $\widetilde{\mathbf{z}}_{n}=\mathbf{U}_{n}^{*} \mathbf{z}_{n}$. As $\widetilde{\mathbf{z}}_{n}$ has the same distribution as $\mathbf{z}_{n}$, the original channel becomes

$$
\widetilde{\mathbf{y}}_{n}=\boldsymbol{\Sigma}_{n} \widetilde{\mathbf{x}}_{n}+\widetilde{\mathbf{z}}_{n} .
$$

Eq. (4) shows that the channel in Eq. (1) can be decomposed into $K$ parallel SISO channels as

$$
\widetilde{y}_{n k}=\gamma_{n k} \widetilde{x}_{n k}+\widetilde{z}_{n k}, \quad k=1,2, \ldots, K .
$$

In the CR system, the transmit power and achievable data rate of the SU's are limited by the interference threshold imposed by the PU. We propose to derive an optimal power with FSA input distributions based on the convex optimization problem. The relationship between MI and MMSE is the key to solve the optimum power allocation problem and is given by [13]

$$
\frac{d I(s n r, S)}{d(s n r)}=m m s e(s n r, S),
$$

where $I($.$) represents MI and S$ denotes an arbitrary input distribution, e.g., M-QAM or Gaussian. We remove $S$ from equations in the rest of the paper, whenever no ambiguity arises. In an ISS scheme, two types of interference, i.e., the one from SU into the PU and vice versa, are introduced to the 


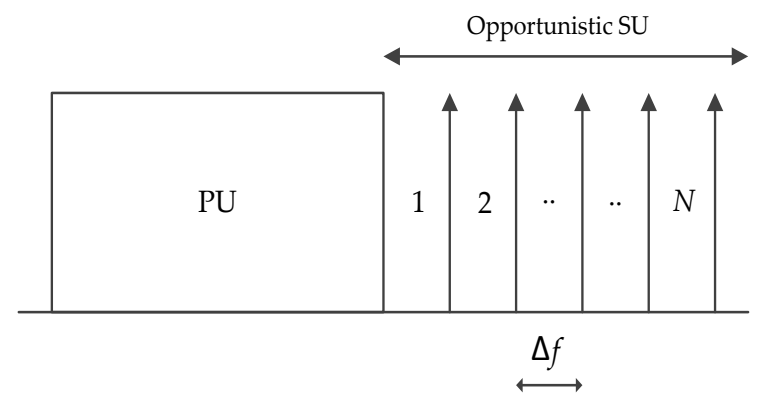

Fig. 2. Co-existence of PU and SU in frequency localized way.

system. Our objective is to protect the PU from unacceptable interference, therefore, we will only consider interference introduced by the SU into the PU band which is given by [7]

$$
J_{n k}\left(d_{n}, p_{n k}\right)=p_{n k} T_{s} \int_{\left(d_{n}-\frac{1}{2}\right) \Delta f}^{\left(d_{n}+\frac{1}{2}\right) \Delta f}\left(\frac{\sin \pi f T_{s}}{\pi f T_{s}}\right)^{2} d f,
$$

where $J_{n k}$ is the interference introduced by the $n$th subcarrier of the SU into PU band at the $k$ th antenna, $T_{s}$ is the symbol duration, $\Delta f$ is the frequency spacing between two adjacent subcarriers and $d_{n}$ represents the spectral distance between the $n$th subcarrier of the SU and PU band.

\section{Optimal Power Allocation Policy for MIMO-OFDM SCHEME}

The objective is to calculate an optimal power with an arbitrary input distribution that maximizes the MI of the SU, provided that the interference introduced into the PUs' band does not exceed a certain level. This problem can be defined as an optimization problem as follows;

$$
\max _{p_{n k}} \sum_{n=1}^{N} \sum_{k=1}^{K} I\left(p_{n k} \gamma_{n k}\right)
$$

subject to

$$
\begin{gathered}
\sum_{n=1}^{N} \sum_{k=1}^{K} J_{n k}\left(d_{n}, p_{n k}\right)=\frac{\tau_{t h}}{\Omega M_{r}} \\
p_{n k} \geq 0, \quad \forall n \in N \quad \forall k \in K,
\end{gathered}
$$

where $N$ and $\tau_{t h}$ represent the total number of available subcarriers and interference threshold prescribed by the PU, respectively. Whereas, $\Omega$ is the path loss and is a function of the distance between the SU transmitter and the PU receiver. Theorem 1: Optimal power with an arbitrary input distribution that maximizes the SU data rate is as follows;

$$
p_{n k}^{\star}= \begin{cases}\frac{1}{\gamma_{n_{k}}} \mathrm{mmse}^{-1}\left(\frac{\lambda \Phi_{n k}}{\gamma_{n_{k}}}\right) & \text { if } \frac{\gamma_{n_{k}}}{\Phi_{n_{k}}}>\lambda, \\ 0 & \text { if } \frac{\gamma_{n_{k}}}{\Phi_{n k}} \leq \lambda,\end{cases}
$$

where $\Phi_{n k}=\frac{\partial J_{n k}}{\partial p_{n k}}=T_{s} \int_{d_{n}-\frac{\Delta f}{2}}^{d_{n}+\frac{\Delta f}{2}}\left(\frac{\sin \pi f T_{s}}{\pi f T_{s}}\right)^{2} d_{f}$ and $\lambda$ is the Lagrange multiplier which can be calculated using numerical methods (such as bisection, secant, or Newton) for solving the following equation

$$
\sum_{n=1}^{N, \frac{\gamma_{n k}}{\Phi_{n k}}>\lambda} \sum_{k=1}^{K} \frac{1}{\gamma_{n k}} \mathrm{mmse}^{-1}\left(\frac{\lambda \Phi_{n k}}{\gamma_{n k}}\right) \Phi_{n k}-\frac{\tau_{t h}}{\Omega M_{r}}=0 .
$$

Proof: As the mutual information is concave [14, section 2.7], the objective function (8) is also concave because the summation preserves the concave function. Also, the constraints (9) and (10) are linear functions of the power. Consequently, the optimization problem is convex [15]. The Slater condition is satisfied with any positive power, $p_{n k}>0$, that satisfies the interference constraint. Therefore, the KKT conditions are necessary and sufficient for the optimal solution. The Lagrangian for the primal problem is as follows;

$$
\begin{aligned}
\mathcal{L}(\mathbf{p}, \lambda, \boldsymbol{\nu})= & -\sum_{n=1}^{N} \sum_{k=1}^{K} I\left(p_{n k} \gamma_{n k}\right)-\nu_{n k} p_{n k} \\
& +\lambda\left(\sum_{n=1}^{N} \sum_{k=1}^{K} J_{n k}\left(d_{n}, p_{n k}\right)-\frac{\tau_{t h}}{\Omega M_{r}}\right) .
\end{aligned}
$$

The KKT conditions are as follows;

Gradient of Lagrangian with respect to $p_{n k}^{\star}$ vanishes:

$$
\begin{gathered}
-\frac{\partial I\left(p_{n k}^{\star} \gamma_{n k}\right)}{\partial p_{n k}^{\star}}+\lambda \underbrace{\frac{\partial J_{n k}}{\partial p_{n k}^{\star}}}_{\Phi_{n k}}-\nu_{n k}=0 . \\
\nu_{n k} \geq 0, \quad p_{n k}^{\star} \geq 0, \quad \lambda \geq 0 . \\
\nu_{n k} p_{n k}^{\star}=0 .
\end{gathered}
$$

Using the fact that $\frac{\partial I\left(p_{n k}^{\star} \gamma_{n k}\right)}{\partial p_{n k}^{\star}}=\gamma_{n k} \operatorname{mmse}\left(p_{n k}^{\star} \gamma_{n k}\right)$, equation (14) can be rewritten as

$$
-\gamma_{n k} \operatorname{mmse}\left(p_{n k}^{\star} \gamma_{n k}\right)+\lambda \Phi_{n k}-\nu_{n k}=0 .
$$

From (15) and (17), we have

$$
\frac{\gamma_{n k}}{\Phi_{n k}} \operatorname{mmse}\left(p_{n k}^{\star} \gamma_{n k}\right) \leq \lambda,
$$

and from (16) and (17), we obtain

$$
p_{n k}^{\star}\left\{\lambda \Phi_{n k}-\gamma_{n k} \operatorname{mmse}\left(p_{n k}^{\star} \gamma_{n k}\right)\right\}=0 .
$$

Consequently, if $p_{n k}^{\star}>0$ then from (19) we obtain $\lambda=$ $\frac{\gamma_{n_{k}}}{\Phi_{n_{k}}} \operatorname{mmse}\left(p_{n k}^{\star} \gamma_{n_{k}}\right)$, therefore

$$
\begin{gathered}
\operatorname{mmse}\left(p_{n k}^{\star} \gamma_{n k}\right)=\frac{\lambda \Phi_{n k}}{\gamma_{n k}}, \\
p_{n k}^{\star}=\frac{1}{\gamma_{n k}} \mathrm{mmse}^{-1}\left(\frac{\lambda \Phi_{n k}}{\gamma_{n k}}\right) .
\end{gathered}
$$

Since mmse $\left(p_{n k}^{\star} \gamma_{n_{k}}\right)<1$ when $p_{n k}^{\star}>0$, we derive from (18) $\frac{\gamma_{n_{k}}}{\Phi_{n_{k}}}>\lambda$. On the other hand, as the $\operatorname{mmse}(0)=1$, if $p_{n k}^{\star}=0$, we have from (18) $\frac{\gamma_{j_{k}}}{\Phi_{n_{k}}} \leq \lambda$. 


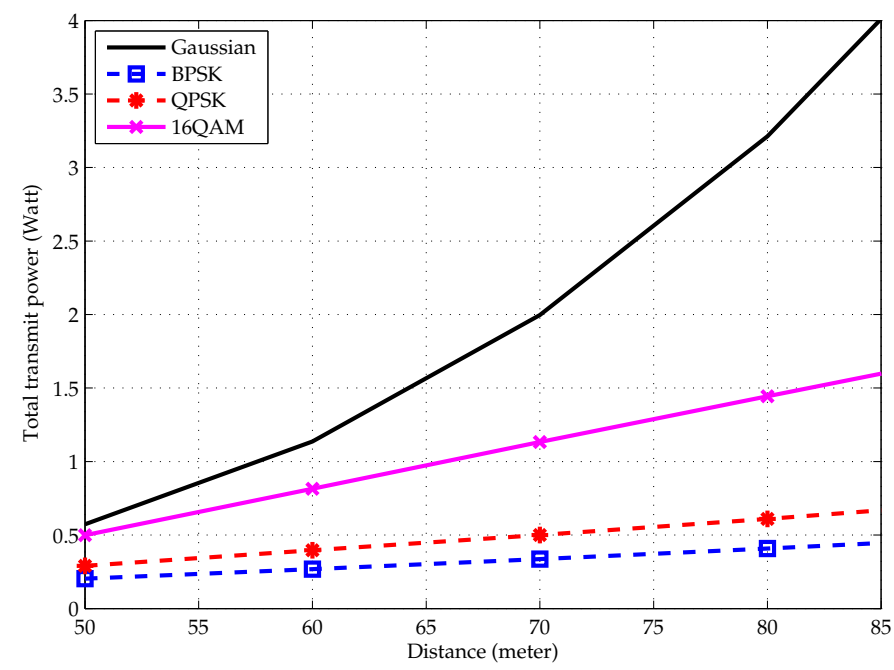

Fig. 3. Optimal power for $2 \times 2 \mathrm{CR}$ system under Gaussian and FSA inputs vs distance.

Note that in [12], the optimal power is derived only for the Gaussian input, whereas, our optimal power derivation is generic and is valid for any input distributions.

\section{Evaluation OF MIMO-OFDM BASED CR SYSTEM}

In this section, we compare optimal power and achievable data rate for the Gaussian and the FSA input through Monte Carlo simulations. The simulations are conducted for a MIMO-OFDM based CR network via an opportunistic scheme as shown in Fig. 2. For practical reasons, we adopt LTE parameters and assume the available bandwidth for the SU transmission is $10 \mathrm{MHz}$ which is divided into 50 resource blocks (RBs) [16]. We consider a simplified path loss model, i.e., $Q\left(\frac{r_{0}}{r}\right)$ [17] for our simulations, where $Q, r_{0}$ and $r$ is constant, reference distance and the distance between the SU transmitter and the PU receiver in meters, respectively. The values of $T_{s}$ and $r_{0}$ are $4 \mu \mathrm{s}$ and 50 meters, and $\tau_{t h}$ is assumed to be equivalent to thermal noise per $\mathrm{RB}$, respectively. The value of $\tau_{t h}$ increases according to $r$ and in our simulation, $r$ ranges from 50 to 85 meters. We further assume the IEEE 802.11 multipath channel model with root mean square delay spread of $50 \mathrm{~ns}$. The results are averaged over 2000 snapshots. We denote the total transmit power $\left(P^{\star}=\sum_{n=1}^{N} \sum_{k=1}^{K} p_{n k}^{\star}\right)$ with Gaussian inputs as $P_{G}^{\star}$ and with FSA inputs as $P_{F}^{\star}$.

In Fig. 3, we compare $P_{G}^{\star}$ and $P_{F}^{\star}$ versus distance for $2 \times 2$ $\mathrm{CR}$ system. We observe from this figure that $P_{G}^{\star}$ is always greater than $P_{F}^{\star}$ over the entire distance range. However, the power difference gap is smaller at lower distance values as compared to higher distance values. The reasons for the power discrepancies are: (i) the increase in $P_{F}^{\star}$ is marginal at higher distance values because MI reaches an upper bound limit, i.e., $\log _{2}|\mathcal{F}|$, where $\mathcal{F}$ denotes the FSA set and $|$.$| represent$ cardinality of the set; (ii) on the other hand, $P_{G}^{\star}$ increases with increasing distance because MI under $P_{G}^{\star}$ has no upper bound limit. It is also observed that, with the same distance

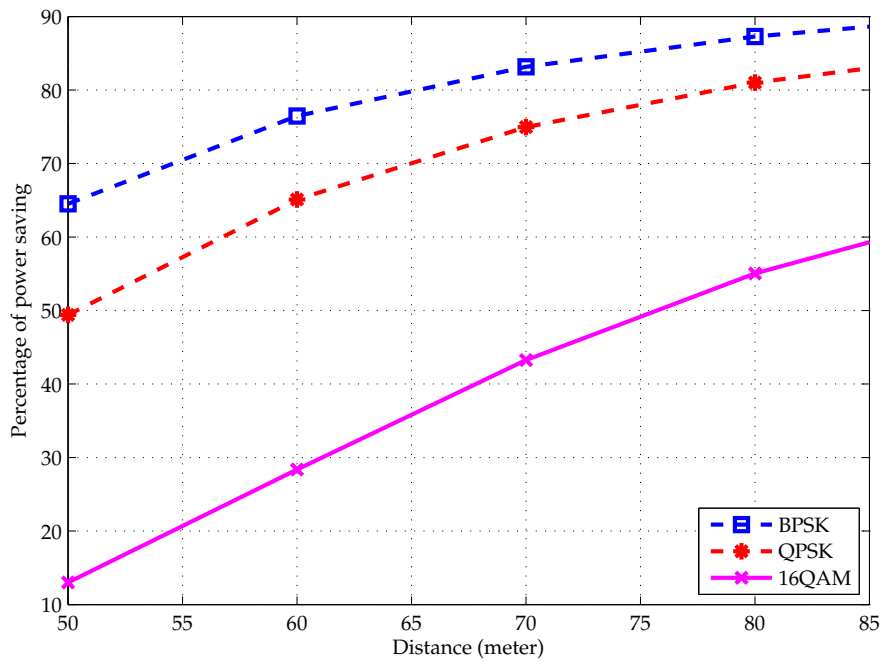

Fig. 4. Percentage of power saving vs distance for $2 \times 2 \mathrm{CR}$ system

value, $P_{F}^{\star}$ increases with increasing modulation scheme, i.e., from BPSK to M-QAM. The power optimality is modulation dependent and thus use of optimality for one modulation scheme if used for another modulation would result in power inefficiency. Hence, for efficient power utilization, power must be optimized according to the actual employed modulation scheme.

We demonstrate the power saving by using our proposed power allocation scheme and compare to the Gaussian scheme in Fig. 4, where we plot percentage power saving = $\frac{P_{G}^{\star}-P_{F}^{\star}}{P_{G}^{\star}} 100 \%$ for BPSK, QPSK and 16-QAM versus distance. From this figure, we observe that there is significant power saving by using the proposed optimal power $P_{F}^{\star}$ compared to $P_{G}^{\star}$. For distance values ranging from $50 \mathrm{~m}$ to $85 \mathrm{~m}$, the transmit power saving is $65-91 \%, 49-87 \%$ and $13-69 \%$ with BPSK, QPSK and 16-QAM inputs, respectively.

Fig. 5 shows a comparison of achieved data rate for the FSA transmission between power optimized for the Gaussian input and the power optimized based on the actual modulation scheme. This shows that the proposed optimal power allocation scheme achieves higher data rate compared to the traditional Gaussian power allocation scheme. In Fig. 6, we show the percentage of rate gain versus distance for $2 \times 2$ $\mathrm{CR}$ system ranging from $50 \mathrm{~m}$ to $85 \mathrm{~m}$; the rate gain is $31.2-18.5 \%, 23.7-16.7 \%$ and $5.6-10.2 \%$ for BPSK, QPSK and 16-QAM inputs, respectively. The justification for this is that, in the CR system where primary and secondary users co-exist in adjacent bands, SU subcarriers which are closer to the PU band cause higher interference, therefore, lower or even zero power can be allocated to these subcarriers. As $P_{G}^{\star}$ is always higher than $P_{F}^{\star}$, it nulls more subcarriers compared to the optimum power for the FSA input and ultimately these subcarriers are wasted. 


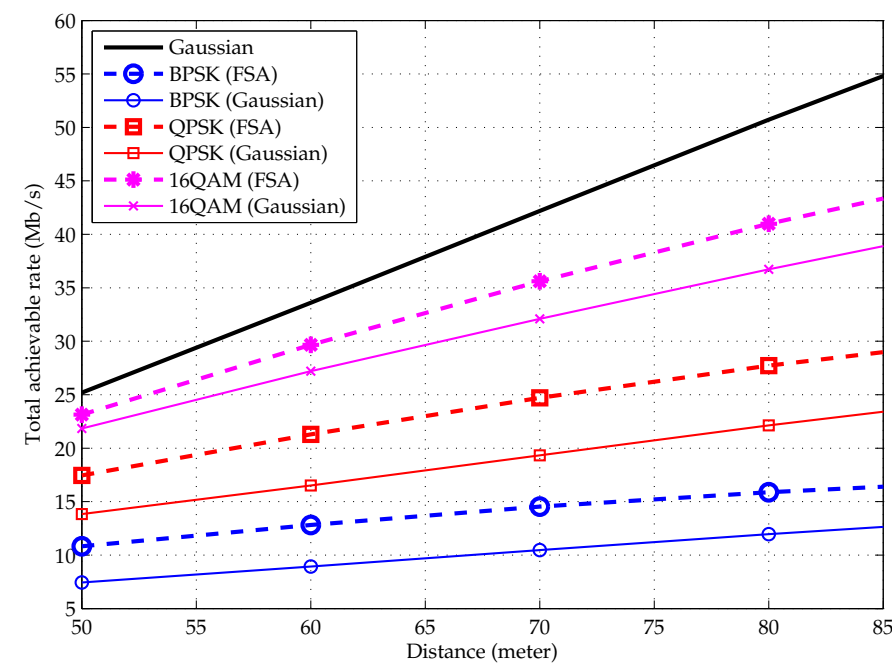

Fig. 5. Achievable data rate under Gaussian and FSA inputs vs distance for $2 \times 2$ CR system

\section{CONCLUSION}

In this paper, we have considered the power allocation problem in MIMO-OFDM based cognitive radio systems under the condition of finite symbol alphabet input distribution applicable to practical systems. The optimal power allocation has been derived by capitalizing on the relationship between mutual information and MMSE using standard convex optimization techniques. The proposed optimal solution for the finite symbol alphabet is evaluated and compared with its conventional counterpart that assumes a Gaussian input. It has been shown via the simulation results that, our proposed scheme significantly outperforms the power allocation based on Gaussian inputs in terms of transmit power saving and achievable data rate. Consequently, system spectrum efficiency and energy efficiency can be improved by using the proposed power allocation scheme. Furthermore, we have shown that as the modulation order increases, the optimal transmit power also increases. Therefore, the power should be optimized based on the employed modulation scheme to achieve a desired energy efficiency.

\section{ACKNOWLEDGMENT}

This work has been supported by the India UK Advance Technology Center of Excellence in Next Generation Networks, Systems and Services (www.iu-atc.com).

\section{REFERENCES}

[1] A. Shukla, "Cognitive radio technology-a study for Ofcom," Tech.Rep. 830000143, QinetiQ Ltd, Hampshire, UK, 2006.

[2] "Federal communications commission: Spectrum policy task force report," http://fjallfoss.fcc.gov, nov. 2002.

[3] A. Sendonaris, E. Erkip, and B. Aazhang, "User cooperation diversity. part I. system description," IEEE Trans. Commun., vol. 51, no. 11, pp. 1927-1938, Nov. 2003.

[4] S. Srinivasa and S. Jafar, "Cognitive radios for dynamic spectrum access - the throughput potential of cognitive radio: A theoretical perspective," IEEE Commun. Mag., vol. 45, no. 5, pp. 73-79, May 2007.

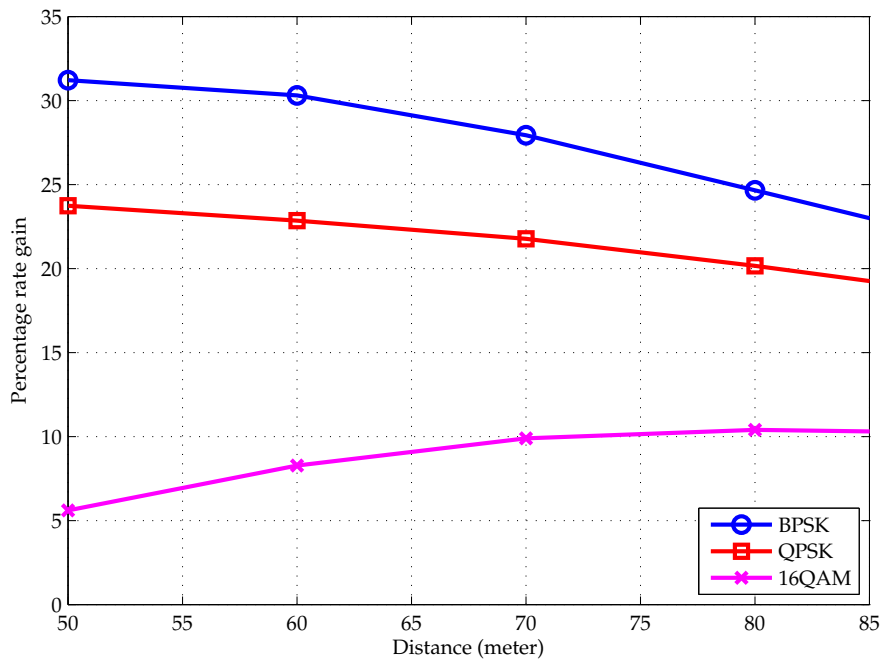

Fig. 6. Percentage of rate gain vs distance for $2 \times 2 \mathrm{CR}$ system.

[5] H. Mahmoud, T. Yucek, and H. Arslan, "OFDM for cognitive radio: merits and challenges," IEEE Wireless Commun., vol. 16, no. 2, pp. 6-15, April 2009.

[6] T. Weiss, J. Hillenbrand, A. Krohn, and F. Jondral, "Mutual interference in OFDM-based spectrum pooling systems," 59th IEEE Vehicular Technology Conference, vol. 4, pp. 1873 - 1877, May 2004.

[7] G. Bansal, M. Hossain, and V. Bhargava, "Optimal and suboptimal power allocation schemes for OFDM-based cognitive radio systems," IEEE Trans. Wireless Commun., vol. 7, no. 11, pp. 4710-4718, Nov. 2008.

[8] Z. Hasan, G. Bansal, E. Hossain, and V. Bhargava, "Energy-efficient power allocation in OFDM-based cognitive radio systems: A risk-return model," IEEE Trans. Wireless Commun., vol. 8, no. 12, pp. 6078-6088, Dec. 2009.

[9] B. Devillers, J. Louveaux, and L. Vandendorpe, "Bit and power allocation for goodput optimization in coded parallel subchannels with ARQ," IEEE Trans. Signal Process., vol. 56, no. 8, pp. 3652-3661, 2008.

[10] A. Lozano, A. Tulino, and S. Verdu, "Optimum power allocation for parallel Gaussian channels with arbitrary input distributions," IEEE Trans. Inf. Theory, vol. 52, no. 7, pp. 3033-3051, July 2006.

[11] A. Sohail, M. Al-Imari, P. Xiao, and B. Evans, "Optimal power allocation for OFDM based cognitive radio systems with arbitrary input distributions," Accepted in IEEE Vehicular Technology Conference, 2013.

[12] H. Shahraki and K. Mohamed-pour, "Power allocation in multipleInput Multiple Output Orthogonal Frequency Division Multiplexingbased cognitive radio networks," IET Commun., vol. 5, no. 3, pp. 362370,2011

[13] D. Guo, S. Shamai, and S. Verdu, "Mutual information and minimum mean-square error in gaussian channels," IEEE Trans. Inf. Theory, vol. 51, no. 4, pp. 1261-1282, April 2005.

[14] T. M. Cover and J. A. Thomas, Elements of Information Theor. Hoboken, New Jersey: Wiley, 2006.

[15] Z.-Q. Luo and W. Yu, "An introduction to convex optimization for communications and signal processing," IEEE J. Sel. Areas Commun., vol. 24, no. 8, pp. 1426-1438, Aug. 2006.

[16] J. Zyren and W. McCoy, "Overview of the 3GPP long term evolution physical layer," Freescale Semiconductor, Inc., white paper, July 2007.

[17] A. Goldsmith, "Wireless communications." New York: Cambridge University Press, 2005. 